\title{
LA ANÁFORA CONCEPTUAL EN EL DISGURSO DE DIVULGACIÓN CIENTÍFICA EN FRANCÉS
}

\author{
GEMMA PEÑa MARTínez \\ Universidad Politécnica de Valencia \\ Mª AmParo Olivares PARdo \\ Universitat de València
}

\section{RESUMEN}

En este artículo pretendemos ofrecer una clasificación de aquellas marcas anafóricas de carácter resumitivo o conceptual, en las que los segmentos anafóricos reformulan el contenido proposicional de determinados fragmentos textuales, ampliando y (re)categorizando dicha información. Se trata de un proceso cognitivo de categorización, donde la realidad que el texto va conformando paulatinamente se reformula conforme a nuestra propia concepción del mundo según nuestras propias experiencias como serie de acciones, sucesos, estados, resultados, procesos, cambios y relaciones entre hechos o acontecimientos, con especial alusión al mundo de la ciencia y el saber como un valor mundialmente compartido. También nociones básicas como el espacio-tiempo o la relación de «meronimia» están presentes en dicha categorización.

Palabras clave: anáfora, cognitivismo, categorización.

\section{AbSTRACT}

In this article we try to offer a classification of the anaphoric marks of resumpitive or conceptual character, in which the anaphoric segments reformulate the propositional content from certain textual fragments, extending and (re)categorizing this information. This is a cognitive process of categorization, where the reality that the text is conforming gradually reformulates on the basis of our own conception of the world according to our own experiences like series of actions, events, states, results, processes, changes and relations between facts or events, with special reference to the world of science and the knowledge like a value world-wide shared. Also basic basic concepts as the space-time or the relation of meronymy is present in this categorisation.

Key Words: anaphora, cognitivism, categorization.

\section{INTRODUCCIÓN}

Este trabajo se centra en los procesos interpretativos y en la construcción del sentido del texto, mundo concreto de referencia con una doble perspectiva de «repetición»y «progresión» (p. ej. continuidad y 
progresión tópicas o temáticas). La realidad se construye y transforma a través del texto que autor y lector ${ }^{1}$ conforman al unísono de forma circular y progresiva. Para garantizar la cohesión y la coherencia textuales, el lenguaje apela a diversos procedimientos, entre ellos y de manera especial, la «anáfora». Este mecanismo supone el punto de inflexión entre el pasado y el futuro de un texto: las «marcas anafóricas» requieren necesariamente para su interpretación la participación de otros segmentos del mismo texto, donde se crea progresivamente una referencia propia concebida en términos de dependencia semántico-pragmática. En los procesos anafóricos influye especialmente un saber compartido transmitido socialmente: el lenguaje no construye una descripción del mundo, sino que enunciador y coenunciador formalizan a través del lenguaje su propia visión del mismo. La realidad extra-lingüística es una variable más, pero no la única: nuestra percepción impone su forma al objeto real, tangible.

En este estudio, hemos recopilado un corpus de textos de especialidad, artículos científico-divulgativos sobre biología, medicina y genética, publicados en la revista francesa La Recherche desde 1996 hasta 2003 en su versión electrónica. Dado su carácter eminentemente explicativo e informativo y puesto que vehiculan contenidos complejos que solicitan una mayor atención tanto por parte del autor como por parte del lector, este tipo de textos requiere una notable cohesión y coherencia discursivas; codificación y descodificación se realizan a través de diversos procesos de retroalimentación discursiva sujetos a una rigidez superior a la de otro tipo de discurso (por ejemplo, literario o poético). En este espacio textual, la construcción del sentido pasa por reformulaciones constantes de la realidad que el texto construye y que el lector almacena en su memoria discursiva.

\section{Nociones Clave}

\subsection{La cohesión}

En los procesos de textualización, las marcas anafóricas constituyen un mecanismo fundamental, ya que expresan las distintas relaciones de cohesión discursiva, noción clave que permite la identificación de un texto como un todo único. Recordaremos que la «cohesión» facilita la interpretación de un elemento discursivo a través de otro componente lingüístico explicitado verbalmente, que lo presupone y permite su correcta descodificación. En este sentido, la «anáfora» asegura la unidad

\footnotetext{
1 Remitimos al «lector activo», co-protagonista junto al responsable de la enunciación, el autor (cf. Lector in fabula, Eco, 1981).
} 
temática y referencial, dando a entender que una misma entidad ha sido objeto de discurso anteriormente; varios elementos remiten a temas o entidades ya introducidos en el mundo discursivo de enunciador y coenunciador. Como exponen Halliday y Hasan 1976, p. 14:

Cohesion as we have said is not a structural relation; hence it is unrestricted by sentence boundaries, and in its most normal form it is simply the presupposition of something that has gone before, whether in the preceding sentence or not. This form of presupposition, pointing back to some previous item, is known as ANAPHORA. What is presupposed anaphorically may be in the sentence immediately preceding, but it may also be in some earlier sentence; $[\ldots]$. Or it may be the whole of some longer passage $[\ldots]$.

\subsection{El antecedente}

Las «marcas anafóricas» dependen de la referencia de otras unidades lingüísticas, el «antecedente», expresión semánticamente autónoma y potencialmente referencial que determinará el sentido concreto, la interpretación y la referencia del segmento anafórico, expresión no autónoma semánticamente. A este propósito, apuntamos la precisión de Cornish 1999, p. 47:

In both cases, the category in terms of which the existing referent is (re-) conceptualized is repeated in the co-text, which is no doubt a way of signalling that the addressee should update his or her discourse-model description, or antecedent, of the entity in question in the manner indicated.

Tanto Lyons 1977 como posteriormente Dik 1997 conciben las expresiones anafóricas como la representación de entidades mentales que se elaboran a través del discurso y no extra-lingüísticamente. Para Apothéloz 1995, son los propios participantes del acto comunicativo y discursivo quienes construyen paulatinamente de forma inter-subjetiva dichos objetos discursivos, aunque no exista una entidad independiente correlativa en el mundo real y fuera del discurso. Por su parte, Ariel 1996, bajo una perspectiva de carácter psico-lingüístico, establece que la particular forma de señalar de la anáfora, según el grado de accesibilidad cognoscitiva del referente intencional o potencial, permite seleccionar un «antecedente» concreto, al que se accede a través de un segmento anafórico específico y de un contexto preciso de interpretación (cf. la «relevancia» de Sperber y Wilson 1986). El antecedente, para Ariel, es una representación mental y no un segmento del cotexto. Al construir el discurso, el enunciador guía mediante expresiones referenciales a su coenunciador; éste último deberá identificar la representación mental correspondiente a dichas expresiones referenciales por medio de procesos inferenciales. 
Recordemos que el contenido informativo de una elocución debe considerarse como un conjunto ordenado y el aporte de nueva información habrá de ser coherente con lo ya existente; de lo contrario, las implicaciones semánticas, las implicaturas conversacionales y las presuposiciones que operan en función del bagaje cultural de enunciador y coenunciador podrían quedar invalidadas. La pertinencia de las representaciones que el lenguaje construye deriva de los efectos cognitivos o contextuales que se producen y que son el resultado de informaciones de carácter inferencial y lingüístico.

\subsection{Saturación, encapsulación, «labelling»}

Las marcas anafóricas, tal y como hemos indicado anteriormente, constituyen expresiones semánticamente incompletas, cuya interpretación viene determinada por el cotexto en base a determinadas relaciones de dependencia. Entre dos elementos discursivos en relación, íntimamente conectados dentro de un texto, el hecho de interpretar un elemento concreto a través de otro provoca en el mismo una «saturación», tal y como expone Corblin 1987, p. 9, lo que puede implicar una cierta supremacía de la «referencia»sobre el «significado».

Sinclair 1993, p. 8 habla también de «encapsulación» como un procedimiento que permite integrar diversos referentes en el texto, poniendo en evidencia una estrategia discursiva de auto-referencia textual: existe una estructura subyacente a todo discurso por la que cada nuevo enunciado hace referencia al anterior y lo encapsula en un único acto referencial.

En el ámbito anglosajón, Francis 1994, pp. 85-86 introduce el concepto de labelling 'etiquetado', proceso que permite enlazar y organizar el discurso por medio de nombres anafóricos que hacen avanzar la argumentación del autor, participando así en la cohesión léxica. Estas etiquetas retrospectivas empaquetan fragmentos de discurso. Reintroducen como conocida una situación descrita previamente en el texto. No se trata de una mera repetición o de un simple sinónimo, sino que pueden representar una o varias cláusulas. Indican al coenunciador la forma en la que han de ser interpretadas, proporcionando el marco de referencia para una argumentación posterior.

La relación entre una etiqueta y la(s) cláusula(s) que reemplaza, esta manera de clasificar nuestra experiencia cultural en formas estereotipadas, supone la codificación de una percepción del mundo compartida; procesamos dichas experiencias por medio de nuestro discurso en entidades nombrables que, aunque similares, no son en absoluto intercambiables. La encapuslación anafórica es un procedimiento por el cual una frase nominal funciona como una paráfrasis que resume una parte 
textual precedente, permitiendo así la creación de un nuevo referente discursivo gracias a información ya conocida que se convierte en argumento de posteriores predicaciones, como apunta Conte 1996, p. 1.

\subsection{Prominencia/ relevancia}

Reichler-Béguelin 1988 y Kleiber 1994 han descrito asimismo la anáfora, bajo una perspectiva cognitiva, como un fenómeno memorístico. Aparece así un nuevo criterio en la identificación de expresiones anafóricas: la prominencia (saillance) del referente dentro del modelo discursivo que enunciador y coenunciador elaboran a lo largo del proceso comunicativo, compartiendo en su memoria discursiva un antecedente presente en cierto sentido en el universo discursivo inmediato; dicho referente queda sobreentendido gracias a su carácter prominente y puede deducirse a partir de aquellos mecanismos inferenciales que nuestro particular universo de creencias nos permite establecer para acceder al mismo, aunque no se encuentre explicitado verbalmente.

La noción de «relevancia» pertinence permitirá establecer, primero, aquellas hipótesis más adecuadas que nos conducirán a la correcta interpretación de la información vehiculada en un contexto concreto, para determinar, en segundo lugar, el referente más apropiado de una marca anafórica.

$\mathrm{La}$ «anáfora» recoge procesos referenciales en el interior mismo del texto (endofóricos) cuya interpretación se establece a partir del espacio lingüístico (cotexto). Dichos procesos se describen como un modo de validación de la información (evocación) almacenada en nuestro saber compartido, representación mental común a enunciador y coenunciador; la «anáfora» re-actualizaría en nuestra memoria discursiva un elemento referido previamente y constituiría la información, variable o constante, objeto de discurso en el texto precedente, de ahí el valor endofórico del que habla Reichler-Béguelin 1988. Así lo muestra Cornish 2002, p. 45:

Under the conception assumed here, anaphora serves to maintain the preexisting attention focus on one or more referents, as well as the situation in which these referents are involved: while deixis is a means of changing the attention focus, by exploiting various key features of the deictic context (speaker, addressee, place and time of utterance).

\subsection{La anáfora}

Dentro de los fenómenos de encadenamiento transfrástico, la «anáfora» forma parte de aquellos procesos de tratamiento de la informa- 
ción que cuentan con el carácter pragmático propio de la producción y comprensión de los procedimientos referenciales, tal y como argumenta Chastain 1975, p. 206. Así, las marcas anafóricas constituyen instrucciones concretas y precisas, revisadas continuamente a lo largo del proceso discursivo, que nos permiten establecer una interpretación concreta y correcta del sentido del mismo. Son interpretadas por enunciador y coenunciador en función del discurso que paulatinamente van elaborando de forma conjunta y dinámica, según indica Cornish 1990.

La anáfora es, pues, un proceso claramente cognitivo, ya que las expresiones indexicales establecen instrucciones tácitas y concretas que operan dentro del modelo mental discursivo, evocado a través del contexto y cotexto, y que de forma cooperativa construyen enunciador y coenunciador. Un procedimiento así dirige el modelo discursivo conjunto, y ha sido diseñado para que los respectivos modelos discursivos de enunciador y coenunciador coincidan en una misma representación.

\section{LA ANÁFORA CONCEPTUAL}

La «anáfora conceptual» o «resumitiva» supone concretamente la reformulación de ideas aparecidas previamente en el texto; retoma no sólo contenidos esenciales dentro del mismo, sino que los amplía y (re)categoriza sin que la interpretación se vea por ello dañada. La «anáfora de dicto» refiere a la información contenida en un fragmento textual y reformula acciones, procesos, estados, propiedades genéricas, etc. $\mathrm{El}$ antecedente comporta generalmente elementos sintagmáticos de mayor magnitud (una frase, un párrafo o todo un fragmento textual) y la marca anafórica condensa, resume, redefine, reinterpreta y sintetiza la idea vehiculada por el antecedente. El lector ha de poner en marcha todo su saber lingüístico y extra-lingüístico (situacional, enciclopédico, etc.) para poder interpretar el texto. Este tipo de marcas anafóricas implica un procedimiento cognitivo en el que entran en juego procesos inferenciales y mecanismos presuposicionales, lo que conlleva cierta dificultad interpretativa, pues el segmento anafórico busca su interpretante a través de fragmentos textuales dispersos y a menudo muy extensos. Así pues, la «anaphore sur énoncé» de Descombes Dénervaud y Jespersen 1992, p. 80 supone la conceptualización no sólo de la aportación informativa del cotexto precedente sino también de la intención argumentativa del locutor.

Como exponen Auricchio, Masseron y Perrin 1992, p. 31, la anáfora resumitiva funciona a distintos niveles: en primer lugar, condensa una estructura predicativa subyacente; en segundo lugar, retoma y relanza información y, por último, refleja la huella de una evaluación u orientación argumentativa. 


\section{EstUdio DE CASOS}

Esta conceptualización de acciones o actividades concretas de la que hemos hablado permite que aparezcan a menudo términos genéricos como activité $\left(1_{1}\right)$ o action $\left(1_{2}\right)$ :

(1) Des chercheurs ont montré chez le singe l'existence de neurones qui déchargent lors de la planification et de l'exécution des mouvements simples et complexes du bras. La partie postérieure du gyrus cingulaire semble concernée non pas par la sélection perceptive, ou la planification du mouvement, mais peut-être par la réorganisation perceptive après un mouvement ou même le contrôle correctif. En effet les neurones du cortex cingulaire postérieur déchargent après les saccades oculaires et leur activité $e_{1}$ dépend des caractéristiques de la saccade précédente. Cette région pourrait donc jouer un rôle dans l'intégration des conséquences de l'action $_{2}$. (LR289)

Al tratarse principalmente de nombres de acción, esta clase de términos proviene en su mayoría de verbos, explicitados, a menudo, en el cotexto precedente, por lo que irán acompañados en ocasiones de los argumentos propios de la estructura verbal. En otras palabras, dichos sintagmas nominales elevan a estatus de referente, o de objeto de discurso, una serie de informaciones presentes en la predicación anterior, lo cual supone un cambio de nivel y una condensación de la información, como señala Péquegnat 1984. Tal y como establece la gramática tradicional para la formación de nuevas palabras, existen tres tipos de derivación: la «derivación impropia», por la que, sin ningún cambio formal, las palabras adquieren nuevas funciones (la flambée); la «derivación propia», la adición de sufijos que modifican la significación del radical (l'élimination) y la «derivación regresiva», cuando se produce el truncamiento de la sílaba final (la mort); éste último es el grupo deverbativo menos abundante en nuestro corpus. Así por ejemplo, en (2), un mismo verbo collecter puede dar lugar a dos nombres: collecte, que indica la acción en sí, y collection (ejemplo 3), el resultado de la misma:

(2) A partir du milieu des années 1980, quelques généticiens ont ainsi commencé à collecter de l'ADN de familles «prédisposées» et à utiliser les outils de la cartographie moléculaire pour rechercher des gènes «du» cancer du sein. [...]. L'identification des deux gènes a rendu possible une pratique de dépistage génétique fondée sur la recherche de mutations dans BRCA 1 et BRCA 2. Initialement murée dans le contexte de la recherche, en lien avec la collecte des familles, cette activité de médecine prédictive a pris de l'ampleur avec Myriad, aujourd'hui en position dominante. (LR329)

En los casos de «derivación propia» (términos formados por sufijación), encontramos diversos grupos. 
a) Nombres derivados mediante -tion (lat. -tio) o-sion (lat. -sio), los más numerosos, generalmente de carácter abstracto: (l’)expresión, (cette) collection, (leur) présentation o (une telle) articulation.

Dentro de este grupo, la determinación se hace generalmente a través del «artículo definido», del «demostrativo», del «posesivo», y en ocasiones, del adjetivo tel.

Aparecen, por lo tanto, términos introducidos por el «artículo definido», que vehicula una presuposición existencial en la que las afirmaciones a propósito del referente a partir de su aparición (circunstancias de evaluación) adquieren especial importancia para poder determinar el antecedente concreto de la marca anafórica. El definido refiere a una entidad reconocible a partir del simple contenido descriptivo del sintagma en el que se inscribe; presupone la existencia y la unicidad, puesto que señala el único referente posible que puede identificar la realidad designada por tal sintagma. Posee una referencia específica, pudiendo designar uno o varios individuos (particular) o el conjunto de una clase de individuos (genérica). Charaudeau 1992, p. 171 lo expone así:

L'article le actualise l'être d'un double point de vue:

- d'une part, il rappelle que l'être nommé est déjà actualisé par rapport à sa classe d'appartenance (valeur anaphorique);

- d'autre part, il indique que l'être nommé doit être considéré comme ayant une ou des particularités qui se trouvent dans le contexte ou la situation.

Otra serie de marcas viene introducida por el «demostrativo», que proporciona un tipo particular de designación, puesto que actualiza una entidad precisando además su presencia efectiva física o contextual (de ahí los usos anafóricos o deícticos del mismo) en el universo discursivo común a enunciador y coenunciador. Dentro de los designadores, este determinante aísla un objeto inscrito en un contexto determinado que requiere una mención precedente. La función demostrativa se ha considerado asimismo una estructura atributiva clasificatoriaatribución de una propiedad a un objeto. Se trata por lo tanto de una «(re-)clasificación». Como indica Corblin 1990, p. 441:

J'ai développé dans Corblin 1985, 1987 une approche des références démonstratives fondée sur l'idée que les démonstratifs n'utilisent pas leur contenu descriptif pour identifier leur référent; celui-ci doit être localisé grâce à un pointage associé sur la situation d'énonciation, et le contenu nominal du démonstratif, s'il existe, est une (re)classification du référent. [...] La différence entre prédication et (re)classification tient à ce que la prédication engage un contenu de jugement qui peut étre asserté, nié, modalisé, etc., alors que la (re)classification est un acte de référence qui conjoint la désignation d'un objet et son assignation par le locuteur à une classe nominale. 
Dado que funciona indexicalmente, a partir de su propio contexto de enunciación, el demostrativo constituye un «designador directo», tal y como argumenta Kleiber 1986, p. 60:

Autre différence, le choix le/ce, on l'aura remarqué, n'est, d'après notre hypothèse, pas possible après toute phrase $p_{1}$ (phrase introductrice). [...] Pour que le soit approprié en reprise, $p_{1}$ doit être tel qu'il fournisse une circonstance d'évaluation pertinente. Il faut que le référent nouvellement introduit puisse dans $p_{1}$ être envisagé comme une entité saisie indirectement. Cela se produit quand $p_{1}$ peut être conçu comme ne servant pas prioritairement à introduire le nouveau référent. S'il en va autrement, c'està-dire si la vertu première de $p_{1}$ est la présentation du nouveau référent, l'enchaînement qu'opère $p_{2}$ ne peut s'effectuer que sur ce référent et ne s'accorde donc guère avec une saisie indirecte. C'est l'adjectif démonstratif qui est à ce moment-là pertinent. On peut aussi l'exprimer en disant que si le référent qu'introduit $p_{1}$ n'est, en quelque sorte, pas prédominant, il peut être repris par $l e$.

El demostrativo posee, por lo tanto, un valor especial y privilegiado en los procesos discursivos, puesto que refiere a un elemento señalándolo de forma particular en la situación de comunicación.

Tal y como hemos indicado anteriormente, el verbo collecter daba lugar a dos nombres: un deverbativo por truncamiento (derivación regresiva) collecte, que retoma la acción (2), y un nombre sufijado, collection, que recoge el resultado de la misma (3):

(3) Or à la même époque cette même idée progressait en France autour du Centre d'étude du polymorphisme humain (CEPH), développé par Jean Dausset pour collecter l'information génétique complète de familles humaines dont on connaissait bien la généalogie. Ayant pris conscience de la valeur du patrimoine que représentait cette collection unique de gènes, un chercheur très actif de son laboratoire, Daniel Cohen, concevait une approche industrielle destinée à séquencer de grands segments du génome. (LR332)

En cuanto a las marcas determinadas por el «posesivo», éste establece una relación de interdependencia entre dos entidades, una de las cuales constituye el polo de referencia de dicha relación que se supone conocido dentro del universo discursivo. Dentro de este sistema aparecen diversas relaciones, según una combinación de "pertenencia» (avoir) o de «actuación» (faire): de naturaleza (de la parte con el todo), de apropiación o adquisición, de localización, de parentesco o relaciones sociales y profesionales; afectiva, de sentimientos, ideas; de caracterización o formas y resultados de obrar, como plantea Charaudeau 1992. Este tipo de relaciones supone una cierta dependencia hacia una de las personas del acto de interlocución o de delocución. Tal y como recogen Riegel, Pellat y Rioul 1994, p. 158: 
Le déterminant possessif est l'équivalent de le [...] de moi, le [...] de toi, etc., toutes formes agrammaticales en français moderne. Autrement dit, il représente la synthèse de deux éléments généralement disjoints du GN: l'article défini et un complément du nom introduit par $d e$ (en l'occurrence un pronom personnel).

(3) Considérant comme acquises les conclusions de T. Engen sur le long terme, d'autres spécialistes ont plutôt cherché à savoir si la mémoire olfactive se distinguait des autres mémoires sensorielles à court terme. Les expériences, là encore peu nombreuses, doivent être interprétées avec prudence, mais il semble bien que les performances de rappel des items d'une liste de stimuli olfactifs présentés quelques minutes auparavant soient peu sensibles à l'ordre de leur présentation, contrairement aux stimuli visuels et auditifs. (LR344)

Respecto a la determinación a través de $t e l$, éste presenta un nombre sin exigir sin embargo una determinación extra-lingüística, proporcionando al sustantivo una cierta apariencia de «identidad». Como muestran Riegel, Pellat y Rioul 1994, p. 162)

Le déterminant $t e l$ joue le rôle d'une proforme qui sert à désigner un référent déterminable mais non autrement déterminé. On l'emploie là où ne conviendrait ni un déterminant indéfini, parce que l'énoncé exige pour être valide que la réalité visée soit bien déterminée, ni un déterminant défini parce que l'énonciateur ne peut ou ne veut communiquer cette détermination $[\ldots]$.

(5) Depuis une vingtaine d'années, en revanche, sous l'influence de la «révolution cognitive», se développe un ensemble de recherches sur la façon dont s'articulent la cognition et la culture humaines. Comprendre les rapports entre mémoire individuelle et collective appelle à l'évidence une telle articulation. (LR344)

b) Nombres derivados por medio de -age (lat. -aticum) que retoman generalmente una serie de objetos de una misma especie, un estado, una acción o el resultado de la misma, como séquençage.

c) Nombres derivados a través de -aison, -ison, -oison (lat. -ationem, -itionem, -otionem), siendo -aison el sufijo más productivo, añadido principalmente a verbos en $-e r$.

Así, en (6), comparaison establece una similitud entre el funcionamiento de la célula y el de una fábrica, desencadenando toda una serie de asociaciones que recurren a la terminología propia de la industria:

(6) Pourquoi compare-t-on la cellule à une usine? Comme dans une usine, de la matière brute entre dans la cellule et des produits manufacturés sont fabriqués. La production est organisée autour de chaînes de montage. Elle emploie des ouvriers spécialisés, les enzymes. De nombreu- 
ses molécules de régulation jouent le rôle de contremaîtres [...]. A ce niveau sont effectués la décoration finale, l'emballage et l'étiquetage du produit manufactur. Celui-ci est ensuite stocké dans des grains de sécrétion. [...] De même que l'on peut bloquer ou ralentir un atelier dans une usine en fonction des besoins, la cellule ajuste le fonctionnement de ses chaînes de montage en fonction des sources de matière première dont elle dispose, des constituants qu'elle a à fabriquer et des commandes qu'elle reçoit du reste de l'organisme. Cependant, $l a$ comparaison avec une usine a ses limites. (LR288)

d) Nombres derivados mediante -ance (lat. -antiam), sufijo añadido generalmente al participio presente verbal, como sureveillance.

e) Nombres derivados por medio de-ement (lat. -amentum), que generalmente suele significar acción y efecto, como el resultativo raccourcissement.

f) Nombres sufijados en -ure (lat. -uram), menos numerosos, que retoman una serie de objetos de una misma especie, una acción o el resultado de la misma, como ouverture.

Las marcas anafóricas pueden reformular el referente como un «proceso» (affaire, développements, enchaînement, histoire u opération) o como un «suceso» (phénomène (7) o événement), un «estado» (fait, generalmente formando parte de expresiones lexicalizadas, choses (8) o situation) o un «resultado» (effet, performance (9) o résultats).

En (7), le (même) genre de posee un semantismo muy débil, por lo que ha de completar su significado expandiéndose en un complemento determinativo que aporta el verdadero sentido:

(7) De fait, il apparaît que certaines lignées de cloportes sont dotées d'un gène masculinisant capable de faire de la résistance. Ce gène $M$ n'est pas situé sur un chromosome sexuel mais sur un chromosome ordinaire (autosome). Dans certains cas, il est capable de réaliser l'opération inverse de Wolbachia, c'est-à-dire de transformer des femelles génétiques WZ en travestis mâles! Il est également capable de bousculer le facteur $\mathrm{f}$ dans la reproduction de femelles $\mathrm{ZZ}+\mathrm{f}$ et d'assurer la présence d'un nombre respectable de mâles chez les petits. En revanche le gène $M$ est incapable d'empêcher l'effet féminisant de Wolbachia, c'est-à-dire chez des travestis infectés. Mais il est alors à l'origine de nombreux types d'intersexués, certains stériles, d'autres fonctionnant comme des femelles et capables de transmettre ce gène. [...] On retrouve le même genre de phénomène chez des insectes. (LR293)

(8) D'autres analyses récentes ont porté cette fois sur certains gènes des chromosomes non sexuels du noyau cellulaire - dont on sait qu'ils représentent une quantité de matériel génétique environ 200000 fois plus importante que la molécule d'ADNmt. Mais lorsque l'on passe à ces gènes nucléaires, qui sont donc à la fois les plus nombreux et portés tant par les hommes que par les femmes, les choses se compli- 
quent en raison des recombinaisons pouvant survenir entre les chromosomes homologues à chaque génération. (LR302)

(9) Une idée commune est que les fonctions cognitives du cerveau sont localisées dans différentes aires cérébrales. L'étude anatomique de l'organisation du système nerveux suggère en outre que les fonctions sensorielles et motrices sont associées à des régions spécifiques du cerveau. [...] D'autant qu'il s'avérait impossible de localiser les traces mnésiques de fonctions supérieures : par exemple, les études de rats placés dans des labyrinthes après prélèvement d'une partie de leur cerveau suggéraient que seule la taille de la partie enlevée, et non son emplacement, déterminait le niveau de la performance. (LR289)

En otros casos, se retoma un cambio, como changements $\left(10_{2}\right)$, modifications, remaniements, remodelage o transformations $\left(10_{1}\right)$ :

(10) Elle a été comparée par certains auteurs au conditionnement pavlovien, à un niveau cellulaire : si une cellule est active en même temps qu'une autre, leur connexion est «renforcée». La plupart des neurobiologistes admettent ainsi que de telles transformations ${ }_{1}$ permettent de stocker des souvenirs dans le cerveau, bien que ce ne soit pas absolument démontré. C'est dans l'hippocampe que la PLT a été mise en évidence pour la première fois. Or, la capacité à obtenir une PLT diminue avec l'âge. Ce serait là le substrat biologique du vieillissement cognitif. Ces changements ${ }_{2}$ sont dépendants de certains récepteurs, appelés NMDA, acteurs essentiels de la PLT. (LR322)

Algunas marcas ponen de manifiesto la relación entre los distintos acontecimientos narrados: associations, combinaison, coopération, corrélation, interactions, liens, rapports o relations.

Encontramos una categoría de términos que poseen un evidente carácter metalingüístico como contexte o métaphore. También encontramos vocablos que reformulan la terminología empleada por la ciencia como rhétorique o nomenclature. Aparecen asimismo marcas que reformulan el argumento de un fragmento textual, como point, question, sujet, thématique o thème o el contenido propio de una argumentación como argument (11), discours o exposé.

(11) Le cœur de la théorie de Medawar est donc constitué par ce principe : le pouvoir de la sélection naturelle diminue inévitablement avec l'âge. Pour appuyer cet argument, Medawar fit appel à une analogie désormais célèbre. Imaginez, expliqua-t-il, une grande population de tubes à essai dans un laboratoire peuplé de scientifiques, très affairés et souvent maladroits. (LR322)

Dentro de esta sub-clase, encontramos asimismo toda una serie de términos que se sirven de los parámetros de la comunicación humana, tales como canal, information o message. Algunos suponen un cruce de 
voces (polifonía): pueden referir a un simple acto dialógico (dialogue o réponse) o retomar varias voces (discussion), a menudo desde el contraste y enfrentamiento (débat o dispute, 12).

(12) Les biotechnologies ne sont pas avares en controverses médiatiques : si les organismes génétiquement modifiés y ont joué les premiers rôles, ils sont talonnés de près par les tentatives récurrentes, aux Etats-Unis et en Europe, de breveter des séquences d'ADN humain, c'est-à-dire de simples fragments de gènes. Les sujets de la dispute sont en partie familiers: des individus, des firmes et des gouvernements échangent des arguments relevant de l'économie politique à propos de ce qui peut constituer des droits de propriété intellectuelle. (LR332)

Otros términos revelan el particular funcionamiento del mundo de la ciencia. Ciertos vocablos refieren a resultados científicos bajo forma de géneros discursivos, como article, comptes rendus (13), dossier, livre o ouvrage.

(13) Dans un passage célèbre, Hanson compare ce que diraient respectivement Tycho Brahe (partisan du géocentrisme) et Kepler (partisan de l'héliocentrisme) sur leur expérience visuelle lorsqu'ils regardent l'un et l'autre le Soleil. Compte tenu des différences entre le contenu de leurs croyances cosmologiques respectives, Tycho Brahe et Kepler rapporteraient leur expérience visuelle en des termes différents. De la différence entre leurs comptes rendus respectifs, Hanson conclut que Tycho Brahe et Kepler ne voient pas la même chose lorsqu'ils regardent le Soleil: l'un voit un objet qui se meut autour de la Terre; l'autre voit un astre immobile au centre du système solaire. (LR309)

O hacen referencia a la experimentación científica y a sus métodos de estudio y comprobación, como découverte, données $\left(14_{1}\right)$, examens, expérience $\left(14_{2}\right)$, exploration, invention, manipulation, technique o tests:

(14) L'idée est séduisante sur le plan intuitif. Elle paraît toutefois en contradiction avec les travaux sur un autre type de mémoire, en apparence très proche: l'apprentissage du «savoir-faire», c'est-à-dire d'habiletés motrices ou perceptives. Cette fois-ci, c'est exactement l'inverse, soit une expansion de la région cérébrale activée, qui a été observée en imagerie. De telles données ${ }_{1}$ ont été publiées l'année dernière par Avi Karni et ses collègues, du NIH à Bethesda. Dans l'expérience ${ }_{2}$, les participants doivent apprendre à pianoter avec les doigts d'une main, en suivant une séquence précise de mouvements. (LR289)

Estos mismos trabajos de laboratorio pueden retomarse por medio de términos más generales como exercice, tâche o travail.

Otras marcas anafóricas suponen una ilustración concreta de un proceso general, como cas (expresión lexicalizada) o exemple. En (15), aparece de forma excepcional la partícula epidíctica -là: 
(15) Chez les animaux, l'embryon est également issu de la reproduction sexuée et de la rencontre des gamètes mâle et femelle, sauf chez certaines espèces dites parthénogénétiques. Ces dernières font l'économie de la reproduction sexuée: l'ovule se divise sans qu'il y ait eu stimulation par le gamète mâle. Le recours à la parthénogenèse est dit «facultatif» chez certaines espèces comme l'abeille (les mâles sont issus de l'ovule non fécondé, les femelles de l'ovule fécondé), mais il est permanent chez d'autres (certains charançons par exemple). Dans ce cas-là, il y a moins de brassage et de diversité génétiques. (LR362)

Algunos vocablos se hacen eco de las coordinadas espacio/tiempo, básicas en la comprensión y ordenación del mundo. Así encontramos marcas que refieren a parámetros temporales, como cycle $\left(16_{2}\right)$, début, délai, étape $\left(16_{1}\right)$, phase o stade:

(16) L'idée avait surgi dans le cerveau du chimiste Kary Mullis dès le printemps 1983. La «recette» se résume ainsi : prendre un fragment d'ADN simple brin et fabriquer son complémentaire à l'aide d'un ADN polymérase, chauffer la double hélice obtenue -les deux brins se séparent-, refroidir, recommencer la synthèse pour obtenir deux doubles hélices, les chauffer pour à nouveau séparer les brins, refroidir, etc. Chaque étape ${ }_{1}$ doublant le nombre de copies de la séquence originale, il était théoriquement possible d'obtenir rapidement des millions de copies du fragment original d'ADN. [...] La mise en œuvre de la technique reste cependant relativement lourde : la polymérase utilisée, issue de la très commune Escherichia coli, est dénaturée à chaque cycle de chauffage $e_{2}$, et il faut recharger la préparation en permanence. (LR317)

Dentro de estas marcas de carácter espacial, cabría distinguir entre el espacio como movimiento o como elemento estático, en ocasiones, altamente estructurado; algunos de estos términos se encuentran en el límite de la «anáfora de dicto» de carácter cognitivo, donde el espacio físico se convierte en espacio mental por el que estructuramos el mundo que nos rodea. Sin embargo, en los términos que ahora recogemos, lo físico y material posee todavía una carga importante. Aparecen términos en los que el espacio se percibe desde su movilidad, como cheminement (17), direction, flux, mouvement, pas o voie.

(17) Il semble exister une autre sous-division de l'AMS pour les mouvements du regard. Si on stimule cette sous-zone par les mouvements des yeux on observe des saccades oculaires qui, au lieu d'avoir une direction fixe, convergent toutes vers un point. On a nommé chez le singe cette aire «champ oculomoteur supplémentaire» (COS). Des patients porteurs de lésions de l'AMS gauche présentent des déficits pour l'exécution de séquences de deux ou trois saccades vers des cibles visuelles mémorisées. Cette région est donc sans doute essentielle pour l'organisation de cecheminement de saccades sur une scène visuelle. Nous 
avons aussi montré, en coopération avec Charles Pierrot-Deseilligny et ses collaborateurs, qu'elle est impliquée dans les saccades mémorisées d'origine vestibulaire. (LR289)

Otros, sin embargo, representan un espacio estático, como angle, base, cadre, carte, cartographie (18), domaine, localisation, position, positionnement, tableau o terrain.

(18) On sous-estime encore sûrement le nombre des aires rétinotopiques. Des régions qui ne répondent pas bien à un stimulus clignotant peuvent nous avoir échappé. D'autres sont peut-être trop petites pour être détectées. Qui plus est, il existe sûrement chez l'homme, comme chez le singe, des aires visuelles non rétinotopiques, mais spécialisées dans la perception et l'interprétation des mouvements des objets, ou de leur forme. Pour compléter la cartographie, il faut donc recourir à des tests qui identifient les aires non par la portion d'espace qu'elles «voient», mais par leur signature fonctionnelle. (LR289)

Dentro de este grupo, algunos términos refieren a un espacio bien organizado y estructurado, como circuit, niveaux, réseau o structure.

En algunos términos, aparece reflejada la relación de «meronimia», básica en la concepción de toda entidad como un todo organizado en partes bien delimitadas, como ensemble, liste, séquence o série.

Algunos nombres operan una clasificación del referente, como catégorie, classes, genre o type.

Otros términos reformulan el referente como una propiedad, como aptitude, capacité, caractère, caractéristiques, propriétés o qualités. Así, entre las marcas que reformulan el antecedente como una característica, encontramos de nuevo casos de derivaciones que tienen como base adjetivos explicitados en el cotexto precedente.

a) Nombres abstractos derivados mediante -ité (lat. -itas), como sensibilité o intensité.

b) Nombres derivados por medio de -ence (lat. -entia), como différence, divergente, présence o absence.

c) Nombres derivados mediante -ie (gr. -i $\alpha$ ), como symétries, pédophagie y homologie.

d) Nombres sufijados mediante -isme (lat. -ismus del gr. -ıбuós), que indican una noción abstracta, como polymorphisme, providentialisme o vitalisme.

(19) L'engendrement du vivant est beaucoup plus étonnant, sinon merveilleux, et peut prendre des formes extrêmement diverses. Elles apporteraient autant de réponses particulières à l'universalité fondatrice de la mort. De même, la mort est la donnée de référence dans 
la célèbre formule de Bichat, «La vie est l'ensemble des fonctions qui résistent à la mort.» En définissant la vie à partir de la mort, il n'y a évidemment plus à s'interroger sur cette dernière.

On trouve là une autre forme de nécessité, qui repose sur des lois physiques. Le vitalisme, auquel se rattache Bichat, oppose aux forces physiques, forces de mort, d'hypothétiques forces vitales qui obéissent à des lois particulières. (LR322)

Otra serie de términos retoman una función, como fonction o rôle. Algunos vocablos reformulan un carácter instrumental, como dispositif o outil. En ocasiones, los términos pueden incidir en la apariencia física, la forma, como forme o morphologie.

Otras expresiones lexicalizadas como façon o manière constituyen en cierta medida un comodín lingüístico. Como indican Auricchio, Masseron, Perrin 1995, p. 44:

Le recensement de quelques AR [anaphore résomptive] fait apparaître quelques constantes dans la structure des AR: un nom qui figure un lieu ou un espace (point, plan, terrain, domaine, forme), un angle de vue (perspective, égard, titre, optique, point de vue) ou bien une circonstance (contexte, but, hypothèse, fin, conditions, manière), ou enfin une modalité plus énonciative (propos, sujet) qui éventuellement fait état de l'avancée du propos (stade, point); le GN démonstratif est précédé d'une préposition qui relie ou localise l'incidence du propos qui suit.

Otros términos retoman un valor en una escala, como biais, degré, index, probabilités, rythme o valeurs.

\section{Conclusiones}

Para nuestro estudio, nos hemos servido de un género textual concreto, el artículo de divulgación científica, ya que, dado que pretende presentar y transmitir conceptos e ideas, los procesos de cohesión y coherencia textual adquieren notable importancia, entre ellos, y especialmente, el uso de marcas anafóricas. En este tipo de discurso, los esquemas argumentativos y expositivos tienen especial relevancia; hemos encontrado así numerosas nominalizaciones y, por lo tanto, múltiples ejemplos de anáforas léxicas.

Este trabajo presenta un doble interés: ampliar el concepto de anáfora y resaltar su utilidad en el discurso científico o académico. Por una parte, la noción de anáfora se ha visto enriquecida. Su función no es simplemente la de resumir información dada previamente, sino que tiene carácter argumentativo e incluso estilístico. Dicho de otra manera, se trata de una encapsulación inteligente. Es un mecanismo versátil que sirve para desempeñar varios objetivos: 
- en ocasiones, retoma sucesos, estados, resultados, procesos e incluso las relaciones que se producen entre ellos;

- en otros casos, reformula el antecedente con un evidente carácter metalingüístico, a semejanza del esquema comunicativo;

- organiza la escritura en base a las coordenadas espacio-temporales físicas propias del texto; es decir, el espacio y el tiempo son tangibles, en contraposición con otras marcas anafóricas de carácter cognitivo, que no tratamos en nuestro estudio. En estas últimas, se produce un paso del espacio físico al mental, sin apenas restos de su primitivo valor (p. ej. méthode);

- establece una relación «meronímica» entre el antecedente y la marca, al identificar el todo organizado en partes bien delimitadas y estructuradas;

- en último lugar, algunas anáforas suponen una clasificación tanto de tipo formal o física como funcional.

Por otra parte, la anáfora resulta especialmente útil si la aplicamos como técnica de escritura académica, ya que refleja un proceso cognitivo inherente a todo acto comunicativo. Este mecanismo permite la progresión y el aporte de información, sintetizando de manera eficiente y competente al evitar la simple repetición o el uso de estructuras sintácticas que sobrecargan el texto.

\section{REFERENCIAS BIBLIOGRÁFICAS}

Apothéloz, D. (1995): «Nominalisations, référents clandestins et anaphores atypiques», TRANEL (Travaux neuchâtelois de linguistique) 23, pp. 43-173.

ARIEL, M. (1996): «Referring expressions and the +/- coreference distinction», en Fretheim, T. y Gundel, J. (eds.), Reference and referent accessibility, Vol. XxxvıII, Ámsterdam, John Benjamins, pp. 15-36.

Auricchio, A.; Masseron, C. y Perrin, C. (1995): «L'anaphore démonstrative à fonction résomptive», Pratiques 85, pp. 27-52.

Charaudeau, P. (1992): Grammaire du sens et de l'expression, París, Hachette-Éducation.

Chastain, Ch. (1975): «Reference and context», en Keith Gunderson, Minnesota Center for Philosophy of Science (eds.), Language, Mind, and Knowledge, Vol. VII, Minnesota Studies in the Philosophy of Science, Minneapolis, University of Minnesota Press, pp. 194-269.

CoIRIER, P. (1999): «Les types de textes: une approche de psychologie cognitive», Linguistica testuale comparativa, Langues Romanes 42, pp. 11-36.

Conte, M.-E. (1996): «Anaphoric encapsulation», en Tasmowski, L. y De Mulder, W. (eds.), Coherence and anaphora, Belgian Journal of Linguistics 10, pp. 1-11.

Corblin, F. (1985): «Les chaînes de référence: analyse linguistique et traitement automatique», Intellectica 5, 1, pp. 123-143. 
—, (1987): Indéfini, défini et démonstratif. Constructions linguistiques de la référence, Ginebra, Droz.

—, (1990): «Démonstratif et nomination», en Morel, M.-A. y Danon-Boileau, L. (dir.), La deixis, París, Presses Universitaires de France, pp. 439-456.

Cornish, F. (1990): «Anaphore pragmatique, référence et modèles du discours», en Kleiber, G. y Tyvaert, J.-E. (dir.), L'anaphore et ses domaines, Recherches Linguistiques 14, París, Klincksieck, pp. 63-80.

-, (1999): Anaphora, Discourse and Understanding, Oxford, Clarendon Press.

—, (2002): «Anaphora: lexico-textual structure, or means for utterance integration within a discourse? A critique of the Functional Grammar account», Linguistics 40, 3, pp. 469-493.

Descombes Dénervaud, M. y JEsPersen, J. (1992): «L'anaphore conceptuelle dans l'argumentation écrite», Pratiques 73, pp. 79-95.

DIK, S. C. (1997): The theory of functional grammar, (Part II: Complex and derived constructions), Berlín [etc.], Mouton De Gruyter.

Eco, U. (1979): Lector in fabula, Milán, Bompiani (trad. esp.: Lector in fabula. La cooperación interpretativa en el texto narrativo, Barcelona, Lumen, 1981).

FrANCIS, G. (1994): «Labelling discourse: an aspect of nominal-group lexical, cohesion», en Coulthard, M. (ed.), Advances in written text analysis, Nueva York, Routledge, pp. 83-101.

Halliday, M. A. K. y Hasan, R. (1976): Cohesion in English, Londres-Nueva York, Longman.

Kleiber, G. (1986): «Pour une explication du paradoxe de la reprise immédiate», Langue Française 72, pp. 54-79.

—, (1994): Anaphores et pronoms, Lovaina, Duculot.

Lyons, J. (1977): Semantics, Cambridge, Cambridge University Press (trad. esp.: Semántica lingüística: una introducción, Barcelona, Paidós, 1997).

PÉQuegnat, C. (1984): «La construction des points de vue dans le raisonnement», en Grize, J.-B. (ed.), Sémiologie du raisonnement, Berna, Peter-Lang, pp. 67-111.

REICHLER-BÉGUELIN, M.-J. (1988): «Anaphore, cataphore et mémoire discursive», Pratiques 57, pp. 15-43.

Riegel, M.; Pellat, J.-C. y Rioul, R. (1994): Grammaire méthodique du français, París, Presses Universitaires de France.

SinCLAir, J. M. (1993): «Written discourse structure», en Sinclair, J. M., Hoey, M. y Fox, G. (eds.), Techniques of description. Spoken and written discourse, Londres, Routledge, pp. 6-31.

SPERBER, D. y Wilson, D. (1986): Relevance: communication and cognition, Oxford, Blackwell (trad. esp.: La relevancia: comunicación y procesos cognitivos, Madrid, Visor, 1994). 